\title{
A Study on Incidence of Enteric Fever among Febrile Patients in a Tertiary Care Hospital of Eastern India with Special Emphasis on Comparative Evaluation of Blood Culture and Serodiagnosis along with Their Antimicrobial Susceptibility and Sociodemographic Profiles
}

\author{
Santanu Pramanik ${ }^{1}$, Bipasa Chakraborty², Gadadhar Mitra ${ }^{3}$, Somnath Dasgupta ${ }^{4}$, Raja Ray ${ }^{5}$ \\ ${ }^{1}$ The University of Burdwan, Burdwan, West Bengal, India. ${ }^{2}$ Department of Microbiology, R G Kar Medical College, \\ Kolkata, West Bengal, India. ${ }^{3}$ Department of Microbiology, Burdwan Medical College, Burdwan, West Bengal, India. \\ ${ }^{4}$ Department of Medicine, Burdwan Medical College, Burdwan, West Bengal, India. ${ }^{5}$ Department of Microbiology, \\ Institute of Post Graduate Medical Education \& Research (IPGMER), Kolkata, West Bengal, India.
}

\section{ABSTRACT}

\section{BACKGROUND}

Enteric fever is an important communicable disease, endemic in India and continues to be major cause of morbidity and mortality. This study was undertaken with the objectives of detecting the incidence of enteric fever, evaluating their sociodemographic profiles, comparative evaluation of sensitivity and specificity of diagnostic modalities like blood culture, Widal test, and Typhidot test in the laboratory diagnosis of enteric fever and to determine the antimicrobial susceptibility patterns.

\section{METHODS}

Blood samples were collected from 145 patients having fever for at least 5 days and symptoms suggestive of enteric fever. Blood culture and identification by standard conventional methods and antimicrobial susceptibility tests using modified KirbyBauer disc diffusion method were performed. Widal and Typhidot tests were done. Sociodemographic data and socioeconomic classification (updated BG Prasad scale) were collected from every patient by standard questionnaires at the time of sample collection. Data analysis was done using GraphPad Prism software.

\section{RESULTS}

Incidence of enteric fever among 145 suspected febrile patients was $36.55 \%$ of which laboratory confirmed culture positive incidence was $18.62 \%$ and probable cases of enteric fever based only on positive serodiagnosis was $17.93 \%$. Isolation rate of Salmonella Typhi and S. Paratyphi A was $17.24 \%$ and $1.38 \%$ respectively. Resistance showed by these isolates were $55.55 \%$ to ampicillin, $29.63 \%$ to chloramphenicol, $44.44 \%$ to cotrimoxazole, $25.92 \%$ to ciprofloxacin and nalidixic acid, $7.41 \%$ to azithromycin, whereas they were $100 \%$ sensitive to ceftriaxone. Multidrug resistant (MDR) Salmonella Typhi was $22.22 \%$. Widal test \& Typhidot seropositivity were $35.86 \%$ and $26.89 \%$ respectively. The sensitivity, specificity, positive predictive value and negative predictive value of Widal test were $96.29 \%$, $77.97 \%, 50 \%$ and $98.92 \%$ respectively and for Typhidot were $100 \%, 89.83 \%$, $69.23 \%$ and $100 \%$ respectively. Though most of the febrile patients were between 13-24 years of age, the burden of enteric fever was maximum in under 12 years age group and it is the only significant risk factor $(p=0.0020)$ associated with enteric fever. Socioeconomic status showed that majority of patients were in the lower and lower middle class (78.62\%).

\section{CONCLUSIONS}

High rate of MDR S. Typhi is alarming and suggests need for better living standards, health education, correct diagnosis and proper treatment as control strategies.

\section{KEY WORDS}

Enteric Fever, Blood Culture, Widal Test, Typhidot, Antimicrobial Susceptibility, MDR Salmonella Typhi
Corresponding Author: Dr. Bipasa Chakraborty, Flat-1B, Anandam Apartment, 107, Garfa Main Road, Kolkata-700075, West Bengal, India. E-mail: bipasa_doc@yahoo.co.in

DOI: $10.14260 / \mathrm{jemds} / 2019 / 809$

Financial or Other Competing Interests: None.

How to Cite This Article:

Pramanik S, Chakraborty B, Mitra G, et al. A study on incidence of enteric fever among febrile patients in a tertiary care hospital of Eastern India with special emphasis on comparative evaluation of blood culture and serodiagnosis along with their antimicrobial susceptibility and sociodemographic profiles. J. Evolution Med. Dent. Sci. 2019;8(50):3734-3738, DOI: $10.14260 /$ jemds/2019/809

Submission 21-10-2019,

Peer Review 19-11-2019,

Acceptance 06-12-2019,

Published 16-12-2019. 


\section{BACKGROUND}

Enteric fever is an important communicable disease prevalent in Eastern India. Advances in public health and hygiene have led to the virtual disappearance of enteric fever from most of the developed countries. It is a sporadic disease in developed countries with occasional point-source epidemics. ${ }^{1}$ But the disease is still endemic in many developing countries like India where it continues to be major cause of morbidity and mortality. ${ }^{2}$ Potential risk factors for enteric fever are taking contaminated food and water, ${ }^{3,4}$ having close contact with recent enteric fever patient, ${ }^{5}$ poor housing admixture of sewage and drinking water supplies during monsoon flooding due to inadequate and modernised sewage system and finally inadequate personal hygiene, ${ }^{6}$ and recent use of broad-spectrum antibiotics. ${ }^{7}$ With changing pattern in clinical presentation of enteric fever in different age groups and large number of culture negative cases due to early inadvertent use of antibiotics, diagnosis of enteric fever became more difficult. In 1948, entry of chloramphenicol revolutionised treatment of enteric fever transforming a severe and fatal disease into a treatable condition. ${ }^{8}$ But the emergence of resistance to chloramphenicol and other antimicrobial agents gradually became a major set back. ${ }^{9}$ However, rising antimicrobial resistance has been associated with increased severity of illness and related complications. Multidrug resistant (MDR) Salmonella Typhi, resistant to chloramphenicol, cotrimoxazole and ampicillin are prevalent in parts of Asia including India and Africa. ${ }^{10}$ Fluoroquinolone resistance is also widespread and now resistance to third generation cephalosporins and azithromycin sporadic cases have emerged. 11 Treating physician is also facing problem with increase in number of drug resistant strains of Salmonella Typhi. So, to improve our ability of the correct diagnosis, we have to give emphasis on diagnostic modalities of better sensitivity and specificity, along with proper validated antimicrobial susceptibility testing technique from the culture isolates. ${ }^{12,13,14}$

So, this study was undertaken with the objectives to detect the incidence of enteric fever, to evaluate the sociodemographic profiles, to do comparative evaluation between blood culture, Widal test, and Typhidot test in the laboratory diagnosis of enteric fever and to determine the antimicrobial susceptibility patterns.

\section{METHODS}

This is a cross-sectional observational study done at Department of Microbiology, Burdwan Medical College and Hospital (BMCH), West Bengal, a tertiary care hospital giving service to patients coming from rural and urban areas. This study was done from March 2015 to August 2016 after obtaining approval from the Institutional Ethical Clearance Committee. Patients who had attended medicine OPD of BMCH with fever for at least 5 days with associated signs and symptoms suggestive of enteric fever were included in this study.

Blood samples were collected aseptically from 145 patients after taking proper informed consent. The required sample size was calculated using the single proportion formula $\mathrm{N}=(\mathrm{Z})^{2} \mathrm{P}(1-\mathrm{P}) / \mathrm{d}^{2}$ by considering expected prevalence $(\mathrm{P})$ of $31 \%, 95 \%$ confidence interval $(\mathrm{Z}=1.96)$ with a $5 \%$ margin of error or precision (d). Also, these 145 cases were selected based on inclusion criteria attending medicine OPD during the study period. Socio-demographic profile data like age, sex, urban-rural distribution, source of drinking water based on pipeline water supply, tube well or well were collected from every patient by standard questionnaires at time of sample collection. The socioeconomic status of every patient was determined based on modified updated BG Prasad scale. ${ }^{15}$ It is an updated income-based scale which is a measure of the social standing and an important factor affecting the health condition of an individual or a family in the society. ${ }^{15}$

For blood culture, blood was collected aseptically from two different sites half an hour apart and immediately introduced into sterile blood culture bottle with brain heart infusion (BHI) broth. For adult patients $5 \mathrm{ml}$ blood was added in $50 \mathrm{ml}$ of BHI broth and for paediatric patients $2 \mathrm{ml}$ blood added in $20 \mathrm{ml}$ BHI broth. The blood culture bottles were immediately sent to Microbiology laboratory and incubated aerobically at $37^{\circ} \mathrm{C}$ till there were signs of turbidity or growth positivity for up to 7 days. All blood culture bottles with turbidity were sub-cultured on xylose-lysinedeoxycholate agar (XLD), deoxycholate citrate agar (DCA) and MacConkey agar plate and incubated aerobically at $37^{\circ} \mathrm{C}$ for 24 hours. Characteristic growth on XLD and DCA were further identified by standard conventional phenotypic methods of identification using biochemical tests. ${ }^{16}$ Isolation of S. Typhi and S. Paratyphi A were confirmed serologically by slide agglutination test with polyvalent $O$ Salmonella group antisera, and serotype specific antisera procured from Difco Laboratories, (Difco-Thermofisher Scientific) USA.

Antimicrobial susceptibility testing was performed on Muller Hinton agar plate using modified Kirby-Bauer disc diffusion method following standard methods. ${ }^{17}$ The antibiotic discs used were ampicillin $(30 \mu \mathrm{g})$, ceftriaxone (30 $\mu \mathrm{g})$, nalidixic acid $(30 \mu \mathrm{g})$, ciprofloxacin $(5 \mu \mathrm{g})$, chloramphenicol $(30 \mu \mathrm{g})$, tetracycline $(30 \mu \mathrm{g})$, trimethoprim/sulfamethoxazole $(1.25 / 23.75 \mu \mathrm{g})$ and azithromycin $(15 \mu \mathrm{g})$, all procured from Himedia Laboratories Pvt Ltd. Interpretation was done as per Clinical and Laboratory Standards Institute (CLSI) guidelines. ${ }^{17}$

Widal tube agglutination test was done using S. Typhi TO and $\mathrm{TH}$ antigens and $S$. Paratyphi $A \mathrm{H}$ and $\mathrm{BH}$ antigens procured from Tulip diagnostics, Tydal test kit. Serum sample was serially diluted by using fresh $0.95 \%$ saline preparation from 1:20 to $1: 640$ dilution for anti $\mathrm{O}$ and anti $\mathrm{H}$ titre separately in Felix and Dreyers tubes. The test was performed following the manufacturer's instructions.

Typhidot test, a rapid dot-enzyme immune assay, which detects IgG and IgM antibodies against a specific $50 \mathrm{kD}$ outer membrane protein (OMP) antigen of Salmonella Typhi was also done. The test was done on serum samples using Salmonella Typhi IgG/IgM Rapid Test (Sensit RAPID), manufactured by Ubio Biotechnology Systems Pvt Ltd. Following the manufacturer's instructions, the results were visually interpreted within 15 minutes.

We defined enteric fever with blood culture confirmed cases for Salmonella Typhi or Salmonella Paratyphi A, and with positive serodiagnosis based on interpretation of Widal 
test and positive Typhidot IgM test. Widal test was considered positive, when titre was $\geq 1$ : 80 for TO and $\geq 1: 160$ for $\mathrm{TH} / \mathrm{AH} / \mathrm{BH}$ or there was four fold rise in titre in paired sera taken after 10-14 days apart. WHO definition of probable/suspected enteric fever is given as fever of at least 3 days, but to increase the diagnostic sensitivity, fever for at least 5 days duration were included in our study. ${ }^{18}$

\section{Statistical Analysis}

Data analysis from questionnaires and laboratory investigations was done using Excel spread sheet (Microsoft Corporation), descriptive biostatistics, \% prevalence, sensitivity, specificity, positive predictive value and negative predictive value and chi square test by Graph Pad Prism version 5.00 (GraphPad software, San Diego, CA, USA). All statistical tests by chi square test were considered significant if the P-value was $\leq 0.05$.

\section{RESULTS}

During the study period total 145 febrile patients with symptoms suggestive of Enteric fever were included. Incidence of enteric fever among these 145 patients was $36.55 \% \quad(n=53)$, of which laboratory confirmed culture positive incidence was $18.62 \%(n=27)$ and probable cases of enteric fever based on positive serodiagnosis where culture showed no growth was $17.93 \%(n=26)$.

\section{Blood Culture and Antimicrobial Susceptibility Test}

From blood culture, 25 Salmonella Typhi and two S. Paratyphi $A$ were isolated. (table-1) Overall resistance shown by these 27 isolates were $55.55 \%$ to ampicillin $(n=15), 29.63 \%$ to chloramphenicol $(n=8), 44.44 \%$ to cotrimoxazole $(n=12)$, $25.92 \%$ to ciprofloxacin and nalidixic acid $(n=7), 7.41 \%$ to azithromycin $(\mathrm{n}=2)$, whereas all 27 isolates $(100 \%)$ were sensitive to ceftriaxone. (figure-1) Among these, six isolates were multidrug resistant (MDR) Salmonella Typhi $(22.22 \%$ prevalence) with resistance to ampicillin, cotrimoxazole (trimethoprim/ sulfamethoxazole) and chloramphenicol.

\section{Widal Tube Agglutination Test}

The serological positivity by Widal test in this study was $35.86 \%(n=52 / 145)$. Whereas blood culture showed only 27 $(18.62 \%)$ febrile patients as culture confirmed cases, with one Widal false negative test result. (table-1) The sensitivity, specificity, positive predictive value (PPV) and negative predictive value (NPV) of Widal test were 96.29\%, 77.97\%, $50 \%$ and $98.92 \%$ respectively as compared with the gold standard test of blood culture. (table-2)

\section{Typhidot Test}

Serodiagnosis by Typhidot IgM was 26.89\% (n=39/145). (table-1) The sensitivity, specificity, PPV and NPV for Typhidot IgM test were 100\%, 89.83\%, 69.23\% and 100\% respectively. (table-2)

\section{Sociodemographic Profiles}

Out of 145 patients, $76(52.41 \%)$ were male and rest 69 $(47.58 \%)$ were female. Age of the participants ranged from 5 years to 60 years and $60 \%$ study population were less than
25 years old $(n=87 / 145)$ with mean age of 23.51 years. The results of enteric fever cases and its association with sociodemographic factors were shown in table 3.

\begin{tabular}{|c|c|c|c|}
\hline $\begin{array}{c}\text { Laboratory } \\
\text { Investigations (n=145) }\end{array}$ & $\begin{array}{c}\text { Organisms } \\
\text { Identified }\end{array}$ & $\begin{array}{c}\text { No. of Positive } \\
\text { Results }\end{array}$ & $\begin{array}{c}\mathbf{\%} \\
\text { Incidence }\end{array}$ \\
\hline \multirow{2}{*}{ Blood culture } & S. Typhi & 25 & $17.24 \%$ \\
\cline { 2 - 4 } & S. Paratyphi $A$ & 2 & $1.38 \%$ \\
\hline \multirow{3}{*}{$\begin{array}{c}\text { Widal tube } \\
\text { agglutination test }\end{array}$} & S. Typhi & $\begin{array}{c}48 \mathrm{TO}(\geq 1: 80)+ \\
\mathrm{TH}(\geq 1: 160)=46, \\
\mathrm{TO}(\geq 1: 80)=2\end{array}$ & $33.10 \%$ \\
\cline { 2 - 4 } & S. Paratyphi $A$ & $\begin{array}{c}4 \mathrm{TO}(\geq 1: 80) \\
+\mathrm{AH}(\geq 1: 160)\end{array}$ & $2.76 \%$ \\
\hline Typhidot IgM test & S. Typhi & 39 & $26.89 \%$ \\
\hline Table 1. Comparison of Incidence of Enteric Fever by Laboratory
\end{tabular}

Diagnosis by Blood Culture, Widal Test, and Typhidot Test
Dite 1. Comparison of Incidence of Enteric Fever by Laboratory

\begin{tabular}{|c|c|c|}
\hline Parameters & Widal Test & Typhidot \\
\hline Sensitivity & $96.29 \%$ & $100 \%$ \\
\hline Specificity & $77.97 \%$ & $89.83 \%$ \\
\hline Positive Predictive Value & $50 \%$ & $69.23 \%$ \\
\hline Negative Predictive Value & $98.92 \%$ & $100 \%$ \\
\hline Table 2. Comparative Evaluation of Widal Test and Typhidot Test as \\
Compared with Blood Culture \\
\hline
\end{tabular}

\begin{tabular}{|c|c|c|c|c|c|c|}
\hline \multicolumn{2}{|c|}{$\begin{array}{c}\text { Socio-Demographic Parameters } \\
\text { Studied }\end{array}$} & 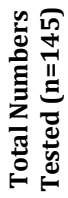 & 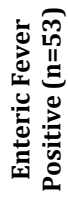 & 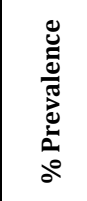 & $\mathbf{X}^{2}$ & $\mathbf{p}$ \\
\hline \multirow[b]{2}{*}{ Sex } & Male & 76 & 33 & $43.42 \%$ & \multirow[b]{2}{*}{3.250} & \multirow[b]{2}{*}{0.0714} \\
\hline & Female & 69 & 20 & $28.98 \%$ & & \\
\hline \multirow{4}{*}{ Age } & $\leq 12$ yrs. & 42 & 25 & $59.52 \%$ & \multirow{4}{*}{14.76} & \multirow{4}{*}{0.0020} \\
\hline & $13-24$ yrs. & 45 & 15 & $33.33 \%$ & & \\
\hline & $25-40$ yrs. & 31 & 7 & $22.58 \%$ & & \\
\hline & $>40$ yrs. & 27 & 6 & $22.22 \%$ & & \\
\hline \multirow{2}{*}{$\begin{array}{l}\text { Urban-rural } \\
\text { distribution }\end{array}$} & Urban & 33 & 11 & $33.33 \%$ & \multirow{2}{*}{0.1908} & \multirow{2}{*}{0.6622} \\
\hline & Rural & 112 & 42 & $37.50 \%$ & & \\
\hline \multirow{3}{*}{$\begin{array}{l}\text { Source of drinking } \\
\text { water }\end{array}$} & Pipeline & 61 & 21 & $34.43 \%$ & \multirow{3}{*}{0.2924} & \multirow{3}{*}{0.8640} \\
\hline & Tube well & 62 & 23 & $37.10 \%$ & & \\
\hline & Well & 4 & 1 & $25 \%$ & & \\
\hline \multirow{5}{*}{$\begin{array}{c}\text { Socio-economic } \\
\text { class } \\
\text { (updated } \\
\text { B G Prasad scale) }\end{array}$} & I (upper) & 4 & 1 & $25 \%$ & \multirow{5}{*}{2.478} & \multirow{5}{*}{0.6486} \\
\hline & II (upper middle) & 6 & 3 & $50 \%$ & & \\
\hline & III (middle) & 21 & 9 & $42.86 \%$ & & \\
\hline & IV (lower middle) & 79 & 25 & $31.65 \%$ & & \\
\hline & $\mathrm{V}$ (lower) & 35 & 15 & $42.85 \%$ & & \\
\hline
\end{tabular}

Table 3. Socio-Demographic Data among Suspected Febrile Patients $(n-145)$ and in Laboratory Diagnosed Enteric Fever Cases $(n=53)$ and their Association

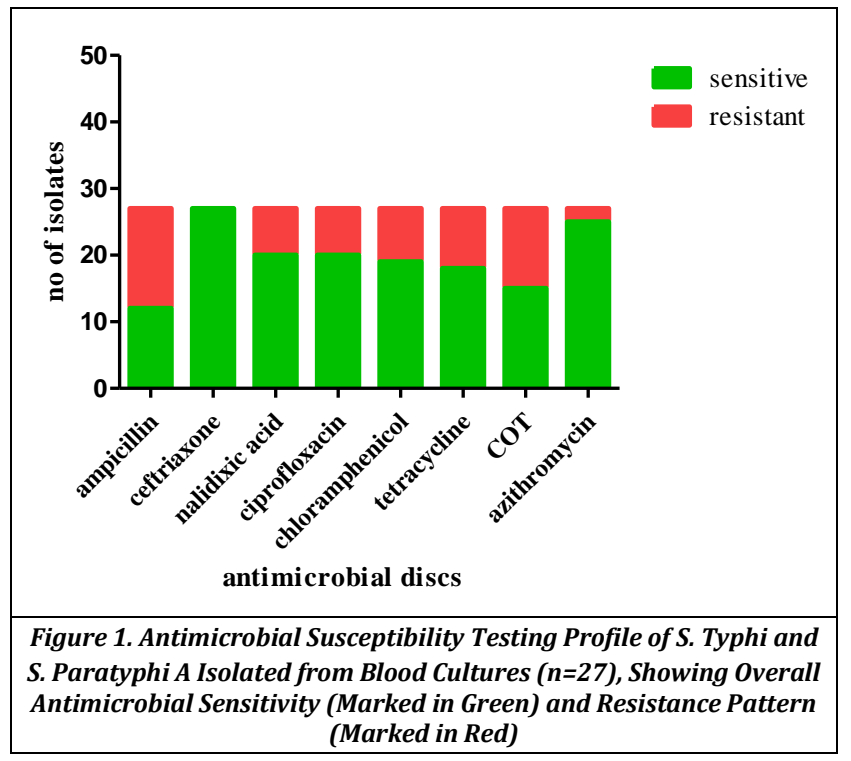




\section{DISCUSSION}

The present study detected higher seropositivity (36.55\%) while lower prevalence from blood culture (18.62\%) with detection of $22 \%$ MDR S. Typhi. MDR S. Typhi and even sporadic cases of azithromycin and quinolone-resistant $S$. Typhi strains have been a major public health concern specially in Pakistan, Sindh reported by Qamar FN et al ${ }^{11}$ and in India ${ }^{19}$ reported by Chowta MN et al. Even there are reports of the emergence and spread of extensively drugresistant (XDR) S. Typhi isolates resistant to chloramphenicol, ampicillin, trimethoprim-sulfamethoxazole, fluoroquinolones, and third-generation cephalosporins, in Pakistan, by Klemm EJ et al, leaving limited treatment options for enteric fever in our hand. ${ }^{20}$ In our study no XDR strains had been isolated but higher rate of isolation of MDR strains was quite alarming. Because of the readily availability of over-the-counter antibiotics and subsequent resistance to these drugs in areas of endemicity, enteric fever is becoming harder to treat. Hence proper AST reporting for each and every case of culture confirmed enteric fever is the need of the hour.

The varied positivity rate between serodiagnosis and blood culture might be due to difference in time of sample collection, false positivity in Widal test and local endemicity of enteric fever with patients having raised antibody titre from old infections. Blood samples when collected beyond 710 days history of fever, had lesser chance to give blood culture positive report, while Widal seropositivity increased. Similarly Widal false negative report with blood culture and Typhidot IgM test positive report was because of duration of fever was 5-7 days. Widal false positive is also not uncommon and could exist because of infection with other Enterobacteriaceae with cross reacting antibody or Malaria. Moreover in our study, Widal test though commonly used as routine screening test, it had low specificity $(77.97 \%)$ and PPV (50\%) in our study indicating high false positive reporting. Similar studies by Chandrashekhar et al ${ }^{21}$ and Aziz $\mathrm{T}$ et al 22 also showed lower sensitivity, specificity, PPV, NPV of Widal tests. Therefore, rapid tests with better sensitivity and specificity are needed for the correct diagnosis of enteric fever.

Typhidot IgM test is a rapid dot-enzyme immune assay that becomes positive in enteric fever as early as in the first week of fever. It had high sensitivity $(100 \%)$ and specificity $(89.83 \%)$ with better performance than Widal test in our study. Similar studies by Bhutta ZA et al, ${ }^{23}$ Sanjeev H et al 24 and Jesudason MV et $\mathrm{al}^{25}$ also showed that Typhidot-M was superior to Widal test in their diagnostic sensitivity and specificity.

Maximum febrile patients were under 13-24 years age group, but the burden of enteric fever was maximum under 12 yrs age group and it is the only significant risk factor associated with prevalence of enteric fever (by Chi-square test, $p=0.0020,95 \%$ CI-Confidence Interval). (table-3) Though no significant association between enteric fever and socioeconomic (SE) status was found in our study (by Chisquare test, $\mathrm{p}=0.6486,95 \% \mathrm{CI}$ ), but majority of patients under this study belonged to lower (V) and lower middle (IV) class ( $\mathrm{n}=114 / 145,78.62 \%)$. Better living standards with good water source, improved sanitation and better hygiene measures along with correct diagnosis and proper treatment may contribute to control endemicity of enteric fever by potentially eliminating faecal carriers and shedders from the population. Also increasing rate of MDR S. Typhi is alarming and suggests need for health education and effective control strategies.

\section{CONCLUSIONS}

High rate of MDR S. Typhi isolation suggests the need for proper AST reporting and judicious use of antibiotics for treating enteric fever. These measures are essential to prevent emergence \& spread of XDR S. Typhi, again transforming enteric fever into a fatal and untreatable condition. Though blood culture is the gold standard test for its diagnosis, the number of culture confirmed enteric fever cases is low. So, we often depend on serodiagnosis. For correct diagnosis of enteric fever, rapid serological tests with better sensitivity and specificity are needed. Also, better living standards, health education, and correct treatment are required to control endemicity of enteric fever.

\section{ACKNOWLEDGEMENT}

The authors thank Dr. Aditya Prasad Sarkar, Associate Professor, Department of Community Medicine, Bankura Sammilani Medical College, Bankura, West Bengal, India for his valuable help in statistical analysis. The authors acknowledge Prof Dr. Sukumar Basak, Ex-Principal of Burdwan Medical College for his support. The authors thank all technical staff and teaching faculties of BMCH.

\section{REFERENCES}

[1] Ackers ML, Puhr ND, Tauxe RV, et al. Laboratory-based surveillance of Salmonella serotype Typhi infections in the United States: antimicrobial resistance on the rise. JAMA 2000;283(20):2668-73.

[2] Kanungo S, Dutta S, Sur D. Epidemiology of typhoid and paratyphoid fever in India. J Infect Dev Ctries 2008;2(6):454-60.

[3] Mermin JH, Villar R, Carpenter J, et al. A massive epidemic of multidrug-resistant typhoid fever in Tajikistan associated with consumption of municipal water. J Infect Dis 1999;179(6):1416-22.

[4] Bhunia R, Hutin Y, Ramakrishnan R, et al. A typhoid fever outbreak in a slum of South Dumdum municipality, West Bengal, India, 2007: evidence for foodborne and waterborne transmission. BMC Public Health 2009;9:115.

[5] Luxemburger C, Chau MC, Mai NL, et al. Risk factors for typhoid fever in the Mekong Delta, southern Viet Nam: a case-control study. Trans $\mathrm{R}$ Soc Trop Med Hyg 2001;95(1):19-23.

[6] Gasem MH, Dolmans WM, Keuter MM, et al. Poor food hygiene and housing as risk factors for typhoid fever in Semarang, Indonesia. Trop Med Int Health 2001;6(6):484-90. 
[7] Luby SP, Faizan MK, Fisher-Hoch SP, et al. Risk factors for typhoid fever in an endemic setting, Karachi, Pakistan. Epidemiol Infect 1998;120(2):129-38.

[8] Woodward TE, Smadel JE, Ley HL Jr, et al. Preliminary report on the beneficial effect of chloromycetin in the treatment of typhoid fever. Ann Intern Med 1948;29(1):131-4.

[9] Mirza SH, Beeching NJ, Hart CA. Multi-drug resistant typhoid: a global problem. J Med Microbiol 1996;44(5):317-9.

[10] Hendriksen RS, Leekitcharoenphon P, Lukjancenko O, et al. Genomic signature of multidrug-resistant Salmonella enterica serovar typhi isolates related to a massive outbreak in Zambia between 2010 and 2012. J Clin Microbiol 2015;53(1):262-72.

[11] Qamar FN, Azmatullah A, Kazi AM, et al. A three-year review of antimicrobial resistance of salmonella enterica serovars Typhi and Paratyphi A in Pakistan. J Infect Dev Ctries 2014;8(8):981-6.

[12] Weyesa JB. Seroprevalence of typhoid fever among subjects with acute febrile manifestations at tertiary care center, Addis Ababa, Ethiopia. Int J Sci Res (IJSR) 2014;3(10):147-55.

[13] Wain J, Hosoglu S. The laboratory diagnosis of enteric fever. J Infect Dev Ctries 2008;2(6):421-5.

[14] Butt T, Ahmad RN, Mahmood A, et al. Ciprofloxacin treatment failure in typhoid fever case, Pakistan. Emerg Infect Dis 2003;9(12):1621-2.

[15] Mangal A, Kumar V, Panesar S, et al. Updated BG Prasad socioeconomic classification, 2014: a commentary. Indian J Public Health 2015;59(1):42-4.

[16] Collee JG, Marmion BP, Fraser AG, et al. Mackie and Mccartney's Practical Medical Microbiology. 14th edn. New York: Churchill Livingstone 1996.
[17] CLSI. Performance Standards for Antimicrobial Susceptibility Testing; Twenty-Fifth Informational Supplement. CLSI document M100-S25. Wayne, PA: Clinical and Laboratory Standards Institute, 2015.

[18] WHO. Background document: the diagnosis, treatment and prevention of typhoid fever. Communicable Disease Surveillance and Response Vaccines and Biologicals, 2003.

[19] Chowta MN, Chowta NK. Study of clinical profile and antibiotic response in typhoid fever. Indian J Med Microbiol 2005;23(2):125-7.

[20] Klemm EJ, Shakoor S, Page AJ, et al. Emergence of an extensively drug-resistant Salmonella enterica serovar Typhi clone harboring a promiscuous plasmid encoding resistance to fluoroquinolones and third-generation cephalosporins. MBio 2018;9(1). pii: :e00105-18.

[21] Chandrashekar, Kumar AYC, Sodhi K, et al. Study of clinical and laboratory profile of enteric fever in pediatric age group. International Journal of Basic and Applied Medical Sciences 2013;3(3):16-23.

[22] Aziz T, Haque SS. Role of Widal test in the diagnosis of typhoid fever in context to other tests. American Journal of Biochemistry 2012; 2(1):p16-18.

[23] Bhutta ZA, Mansurali N, Rapid serologic diagnosis of pediatric typhoid fever in an endemic area: a prospective comparative evaluation of two DOT-enzyme immune assays and the Widal test. Am J Trop Med Hyg 1999;61(4):654-7.

[24] Sanjeev H, Nayak S, Asha PKB, et al. Systematic evaluation of rapid dot-eia, blood culture and Widal test in the diagnosis of typhoid fever. Nitte university Journal of Health Sciences 2013;3(1):21-4.

[25] Jesudason MV, Sivakumar S. Prospective evaluation of a rapid diagnostic test Typhidot for typhoid fever. Ind J Med Res 2006;123(4):513-6. 